\title{
Dust in the bright supernova remnant N49 in the LMC ${ }^{\star}$
}

\author{
M. Otsuka ${ }^{1}$, J. Th. van Loon ${ }^{2}$, K. S. Long ${ }^{1}$, M. Meixner ${ }^{1}$, M. Matsuura ${ }^{3}$, W. T. Reach ${ }^{4,5}$, J. Roman-Duval ${ }^{1}$, \\ K. Gordon ${ }^{1}$, M. Sauvage ${ }^{6}$, S. Hony ${ }^{6}$, K. Misselt ${ }^{7}$, C. Engelbracht ${ }^{7}$, P. Panuzzo ${ }^{6}$, K. Okumura ${ }^{6}$, P. M. Woods ${ }^{8}$, \\ F. Kemper ${ }^{8}$, and G. C. Sloan ${ }^{9}$ \\ (Affiliations can be found after the references)
}

Received 31 March 2010 / Accepted 30 Avril 2010

\begin{abstract}
We investigate the dust associated with the supernova remnant (SNR) N49 in the Large Magellanic Cloud (LMC) as observed with the Herschel Space Observatory. N49 is unusually bright because of an interaction with a molecular cloud along its eastern edge. We have used PACS and SPIRE to measure the far IR flux densities of the entire SNR and of a bright region on the eastern edge of the SNR where the SNR shock is encountering the molecular cloud. Using these fluxes supplemented with archival data at shorter wavelengths, we estimate the dust mass associated with N49 to be about $10 M_{\odot}$. The bulk of the dust in our simple two-component model has a temperature of 20-30 K, similar to that of nearby molecular clouds. Unfortunately, as a result of the limited angular resolution of Herschel at the wavelengths sampled with SPIRE, the uncertainties are fairly large. Assuming this estimate of the dust mass associated with the SNR is approximately correct, it is probable that most of the dust in the SNR arises from regions where the shock speed is too low to produce significant X-ray emission. The total amount of warm $50-60 \mathrm{~K}$ dust is $\sim 0.1$ or $0.4 M_{\odot}$, depending on whether the dust is modeled in terms of carbonaceous or silicate grains. This provides a firm lower limit to the amount of shock heated dust in N49.
\end{abstract}

Key words. ISM: supernova remnants - dust, extinction - submillimeter: ISM - Magellanic Clouds

\section{Introduction}

The source N49 is a bright X-ray and optical supernova remnant (SNR) in the Large Magellanic Cloud (Long et al. 1981), associated with a soft gamma ray repeater (Cline et al. 1982) and unresolved X-ray point source (Rothschild et al. 1994). It has an asymmetric surface-brightness distribution at essentially all wavelengths, which is due to an interaction with a molecular cloud that is located on the southeast limb of the SNR (Vancura et al. 1992; Banas et al. 1997). Based on a simple Sedov model for the SNR, Hughes et al. (1998) concluded that the swept-up mass was the order of $200 M_{\odot}$. Park et al. (2003) recently conducted a detailed study of its X-ray properties using Chandra, confirming that the SNR is relatively young, on the order of $6000 \mathrm{yrs}$. They find that although most of the emission is dominated by interstellar material (ISM), the Chandra observations appear to show "explosion fragments" on the SW side of the SNR in X-rays. According to Bilikova et al. (2007), the X-ray temperature $\left(\sim 1.2 \times 10^{7} \mathrm{~K}\right)$ implies a shock velocity of $920 \mathrm{~km} \mathrm{~s}^{-1}$, while the optical filaments which reflect shocks traversing denser gas have typical line-of-sight velocities of $250 \mathrm{~km} \mathrm{~s}^{-1}$. Hill et al. (1995) estimated the mass of the progenitor of $\mathrm{N} 49$ to be about $20 M_{\odot}$ based on the association with the 10 Myr old association LH 53. A $1720 \mathrm{MHz}$ OH maser emission comes from a region toward the southwest. The remnant N49 is almost certainly the result of a type II explosion.

Apparently first detected at mid-IR wavelengths with IRAS (Graham et al. 1987), the first mid-IR images of the SNR were

* Herschel is an ESA space observatory with science instruments provided by European-led Principal Investigator consortia and with important participation from NASA. obtained with Spitzer (Williams et al. 2006a,b), where N49 is visible not only in the MIPS 24 and $70 \mu \mathrm{m}$ bands, but also in the IRAC 3.6, 4.5, 5.6 and $8 \mu \mathrm{m}$ bands. IRS spectra of the eastern limb show strong line emission from $\mathrm{O}, \mathrm{Ne}, \mathrm{Ar}, \mathrm{Si}, \mathrm{S}$, and $\mathrm{Fe}$ and $\mathrm{H}_{2}$ in the 5-37 $\mu \mathrm{m}$ band (Williams et al. 2006a,b). While lines and dust emission compete at the short wavelengths, van Loon et al. (2010) showed with the low resolution MIPS spectra of the bright blob on the eastern limb that dust is the dominant source of emission at $70 \mu \mathrm{m}$, and estimated a dust mass for N49 of $\sim 0.2 M_{\odot}$.

Dust in SNRs can arise either from the SN itself or from the ISM. Ejecta dust is thought to play a key role as a coolant in the formation of high-redshift galaxies (see, e.g. Morgan \& Edwards 2003). However, given the amount of swept-up material in N49, most of the dust in N49 is expected to be of interstellar origin.

Here we describe observations of N49 obtained with the Herschel Space Observatory. Our primary purpose is to explore the properties of dust in N49, and to obtain a better estimate of the dust mass than was heretofore possible by probing the longer wavelength bands, where larger dust grains are expected to be more apparent. Combining Herschel data and data obtained from other observatories, we construct spectral energy distributions (SEDs) of the entire SNR and of a bright blob on the eastern limb, where the SNR is interacting with a molecular cloud. (see, e.g. van Loon et al. 2010). We use a simple two-temperature model of the SED to estimate dust masses and temperatures.

\section{Observations and data reduction}

The SNR N49 was observed with Herschel as part of the science demonstration program associated with the HERITAGE 


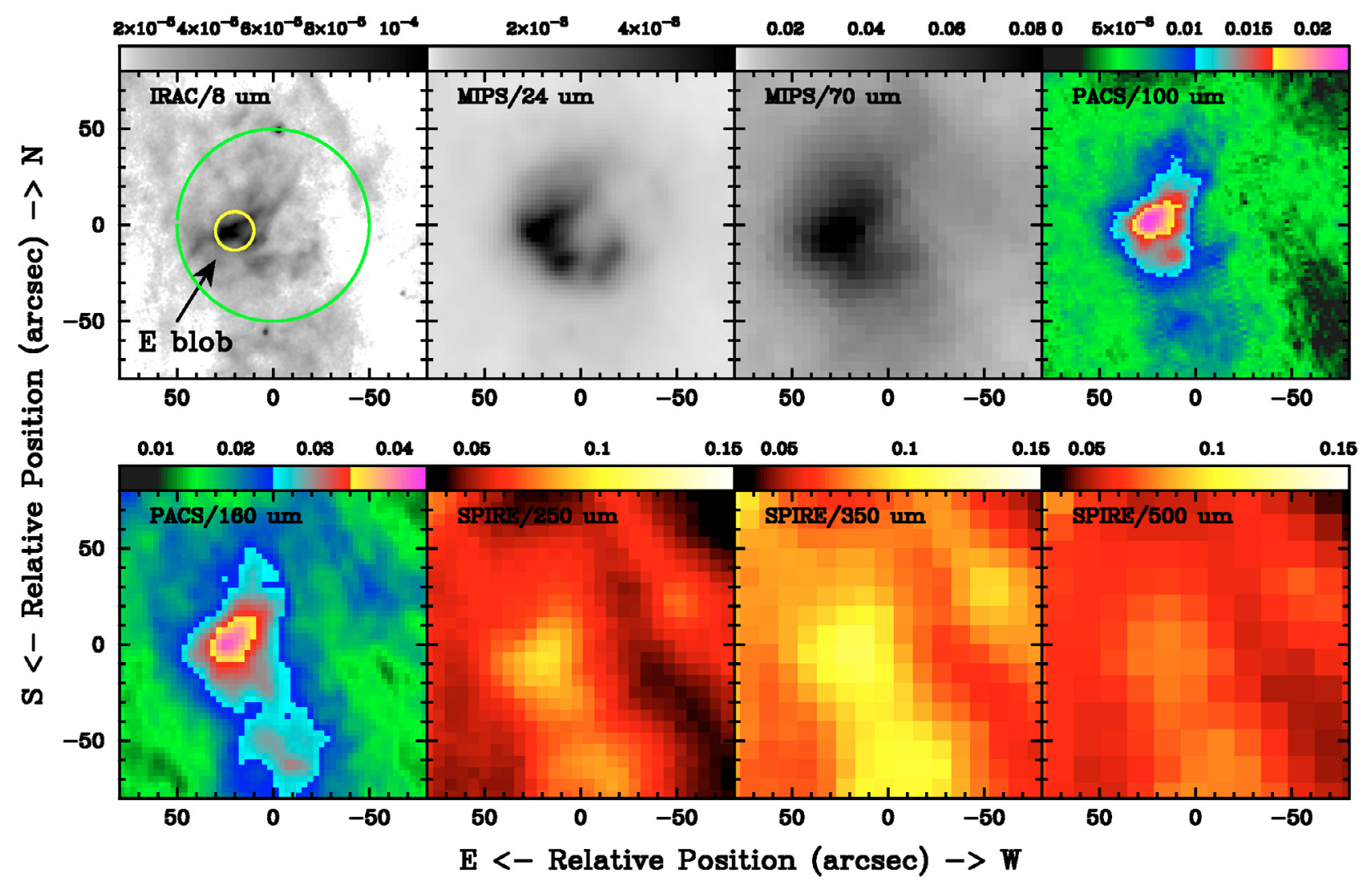

Fig. 1. Selected images of the SNR N49. The intensity is in Jy per pixel. The location of the eastern blob (E blob) is indicated by the arrow. The radii of the green and yellow circles are 50" and 10" when adopting aperture photometry for the whole SNR and the E blob.

program (PI: M. Meixner). The observations and the data reduction process for the PACS green and red bands $(100 / 160 \mu \mathrm{m})$ and SPIRE all bands $(250 / 350 / 500 \mu \mathrm{m})$ are described by Meixner et al. (2010).

To complement the Herschel data, we used archival Spitzer/IRS low- and high-resolution spectra (5-40 $\mu \mathrm{m}$; PI: Gehrz) of the eastern blob (E blob, hereafter), Spitzer/IRAC 3.6/4.5/5.8/8.0 $\mu \mathrm{m}$ and MIPS $24 / 70 \mu \mathrm{m}$ images (Meixner et al. 2006), and the published 3/7/11/15/24 $\mu$ m fluxes for whole SNR (Seok et al. 2008) taken by the AKARI/IRC (Murakami et al. 2007; Onaka et al. 2007). We indicate the position of the E blob in Fig. 1. We rescaled the flux density of the IRS spectrum by a factor of 1.25 in order to match the total flux between 20.34 and $29.46 \mu \mathrm{m}$ of the IRS spectrum and the MIPS $24 \mu \mathrm{m}$ flux. We used the reductions of the IRS and the MIPS spectra $(52-93 \mu \mathrm{m})$ of the E blob made by Kemper et al. (2010) as part the SAGESpec project. The MIPS spectrum has been described in detail by van Loon et al. (2010).

Selected Spitzer and Herschel images are presented in Fig. 1. Although the angular resolution of PACS at 100 and $160 \mu \mathrm{m}$ is comparable to that of MIPS 24 and $70 \mu \mathrm{m}$, the resolution of SPIRE is such that it becomes increasingly difficult to distinguish the SNR from the surrounding molecular clouds. The intensity of the E blob becomes comparable to the southern molecular clouds at longer wavelengths. There is no obvious difference however in the morphology of the SNR as a function of wavelength, although at long wavelengths, where the resolution is poorer, it becomes increasingly difficult to pick out the shell of the SNR. The general interpretation that the SNR is interacting with a molecular cloud on the SE is confirmed by the Herschel images.

To estimate flux densities, we performed aperture photometry. We used $50^{\prime \prime}$ and $10^{\prime \prime}$ radius regions to define the entire
SNR and the eastern blob, respectively (see Fig. 1). We subtracted background from an annulus centered on the SNR with inner and outer radii of $110^{\prime \prime}$ and $130^{\prime \prime}$. In the IRAC $8 \mu \mathrm{m}$ image of Fig. 1, the two regions are indicated as green $\left(50^{\prime \prime}\right.$ radius) and yellow (10") circles. We performed aperture corrections using observed or theoretical point-spread functions. The measured fluxes are listed in Table 1 . The absolute flux calibration is 2 and 5\% for MIPS 24 and $70 \mu \mathrm{m}$ bands and is $20 \%$ for PACS (Poglitsch et al. 2010) and 15\% for SPIRE (Swinyard et al. 2010), respectively. We adopted these values as the flux density uncertainty for these bands. We did not include uncertainties associated with the subtraction of background, but these are at least as large in the SPIRE bands as the uncertainty in the flux calibration. For the other bands, the uncertainty in Table 1 corresponds to the standard deviation of the background.

The SEDs are presented in Fig. 1 for the entire SNR and for the E-blob alone. There are no obvious differences aside from normalization in the two spectra. Williams et al. (2006a,b), based on an early reduction of the IRS spectrum, argued that the emission lines rather than the dust continuum dominate the flux in the MIPS $24 \mu \mathrm{m}$ band. However, our improved reduction of the IRS spectrum shows an obvious dust continuum. Our estimate based on the full $9.7 \mu \mathrm{m}$ width of the MIPS $24 \mu \mathrm{m}$ band is that emission lines contribute about $38 \%$ of the flux. The fluxes plotted at $24 \mu \mathrm{m}$ in Fig. 1 represent the continuum flux, assuming that the line contribution is the same for the entire SNR as for the region covered by the IRS spectrum. At wavelengths of $\lambda \sim 70 \mu \mathrm{m}$ and longer, the radiation from dust grains in the SNR is the main luminosity source. van Loon et al. (2010) found dominant [O I] $63 \mu \mathrm{m}$ line in the MIPS $70 \mu \mathrm{m}$ band to contribute only $11 \%$ of the total emission in that band. Furthermore, based on this, the expected [O I] $146 \mu \mathrm{m}\left({ }^{3} P_{1}-{ }^{3} P_{0}\right)$ flux is $3.4-$ $7.2 \times 10^{-13} \mathrm{erg} \mathrm{s}^{-1} \mathrm{~cm}^{-2}$ when assuming the electron temperature 
Table 1. Flux density of N49.

\begin{tabular}{rccc}
\hline \hline$\lambda$ & \multicolumn{2}{c}{$F_{v}(\mathrm{Jy})$} & \\
\cline { 2 - 3 }$(\mu \mathrm{m})$ & whole SNR & E blob & source \\
\hline 3.2 & $(3.6 \pm 0.4) \times 10^{-2}$ & $\ldots$ & $(1)$ \\
3.6 & $(3.8 \pm 0.6) \times 10^{-2}$ & $(6.0 \pm 0.8) \times 10^{-3}$ & $(2)$ \\
4.5 & $(3.2 \pm 0.5) \times 10^{-2}$ & $(7.4 \pm 0.8) \times 10^{-3}$ & $(2)$ \\
5.8 & $(1.8 \pm 0.9) \times 10^{-1}$ & $(3.0 \pm 0.4) \times 10^{-2}$ & $(2)$ \\
7.0 & $(2.8 \pm 0.3) \times 10^{-1}$ & $\ldots$ & $(1)$ \\
8.0 & $(2.3 \pm 1.0) \times 10^{-1}$ & $(4.1 \pm 0.5) \times 10^{-2}$ & $(2)$ \\
11 & $(3.3 \pm 0.3) \times 10^{-1}$ & $\ldots$ & $(1)$ \\
15 & $(8.7 \pm 0.9) \times 10^{-1}$ & $\ldots$ & $(1)$ \\
24 & $1.6 \pm 0.1$ & $(30 \pm 0.6) \times 10^{-2}$ & $(2)$ \\
70 & $9.9 \pm 0.5$ & $(18 \pm 0.9) \times 10^{-1}$ & $(2)$ \\
100 & $8.5 \pm 1.7$ & $1.4 \pm 0.4$ & $(3)$ \\
160 & $7.2 \pm 1.4$ & $1.2 \pm 0.2$ & $(3)$ \\
250 & $2.1 \pm 0.3$ & $(5.3 \pm 0.8) \times 10^{-1}$ & $(3)$ \\
350 & $(5.0 \pm 0.8) \times 10^{-1}$ & $(2.8 \pm 0.4) \times 10^{-1}$ & $(3)$ \\
500 & $(1.0 \pm 0.2) \times 10^{-1}$ & $\ldots$ & $(3)$ \\
\hline
\end{tabular}

Notes. (In the last column) (1): AKARI (Seok et al. 2008); (2): Spitzer (present work); (3): Herschel (present work).

to be $10^{4} \mathrm{~K}$ and a density $10-10^{4} \mathrm{~cm}^{-3}$. The contribution of [O I] $146 \mu \mathrm{m}$ in the PACS $160 \mu \mathrm{m}$ band is $\sim 7 \%$.

\section{Dust mass in the $\mathbf{N} 49$ system and discussion}

We estimated the dust mass with the line-contribution-subtracted data and its temperature through SED fitting. First, we estimated the contribution of the synchrotron emission to the midto far-IR region. Dickel \& Milne (1998) measured the flux densities at $3.5 / 6 / 13 / 20 \mathrm{~cm}$ for the whole SNR. By extrapolating the flux densities at this radio wavelength to the mid-IR region, we found that the contribution is negligibly small $(<2 \%)$ to the $24 / 70 / 100 / 160 / 250 \mu \mathrm{m}$ flux densities. In the SPIRE $350 / 500 \mu \mathrm{m}$ bands, the contribution is large; $\sim 14 \%$ for $350 \mu \mathrm{m}$ and $\sim 79 \%$ for $500 \mu \mathrm{m}$. Before fitting, we subtracted the contribution of the synchrotron emission to the $350 \mu$ m flux density for the whole SNR and $\mathrm{E}$ blob, assuming that the contribution can be also applied to the E blob. In the fittings we excluded $500 \mu \mathrm{m}$ data.

When we assume optically thin thermal radiation at a dust temperature $T_{\mathrm{d}, i}$ of a component $i$ and a single dust species with the radius $a$, the observed flux density $F_{v}$ is written by

$F_{v}=\left(\frac{4}{3} a \rho D^{2}\right)^{-1} \times \sum_{i} M_{\mathrm{d}, i} B_{v}\left(T_{\mathrm{d}, i}\right) Q_{v, i}$,

where $M_{\mathrm{d}, i}$ is the dust mass of the component $i, B_{v}\left(T_{\mathrm{d}, i}\right)$ is the Planck function of $T_{\mathrm{d}, i}$, and $Q_{v, i}$ is the absorption efficiency, $\rho$ is the dust grain density and $D$ is a distance to the observed dust from us (Kwok 2007). Here, we adopted $D=50 \mathrm{kpc}$ (Schaefer 2008). We considered two types of grains, amorphous carbon and silicates. For amorphous carbon the optical constants were taken from Zubko et al. (1996). We adopted $\rho=2.26 \mathrm{~g} \mathrm{~cm}^{-3}$. To simplify the model, we assume $a=0.1 \mu \mathrm{m}$ uniform grain radius. Next, we considered astronomical silicate only. The optical constants were taken from Draine \& Lee (1984) and a $\rho$ of $3.6 \mathrm{~g} \mathrm{~cm}^{-3}$ was adopted. Due to the lower absorption efficiency at $>70 \mu \mathrm{m}$ than amorphous carbon, a larger dust mass would be estimated. The dust mass estimated for the silicate-only case would be an upper limit. In the fittings we found the best fit $M_{\mathrm{d}, i}$ and $T_{\mathrm{d}, i}$ to the observations by least-square technique. In this process, $M_{\mathrm{d}, i}$ and $T_{\mathrm{d}, i}$ are the free parameters, and all the data are equal of weight.

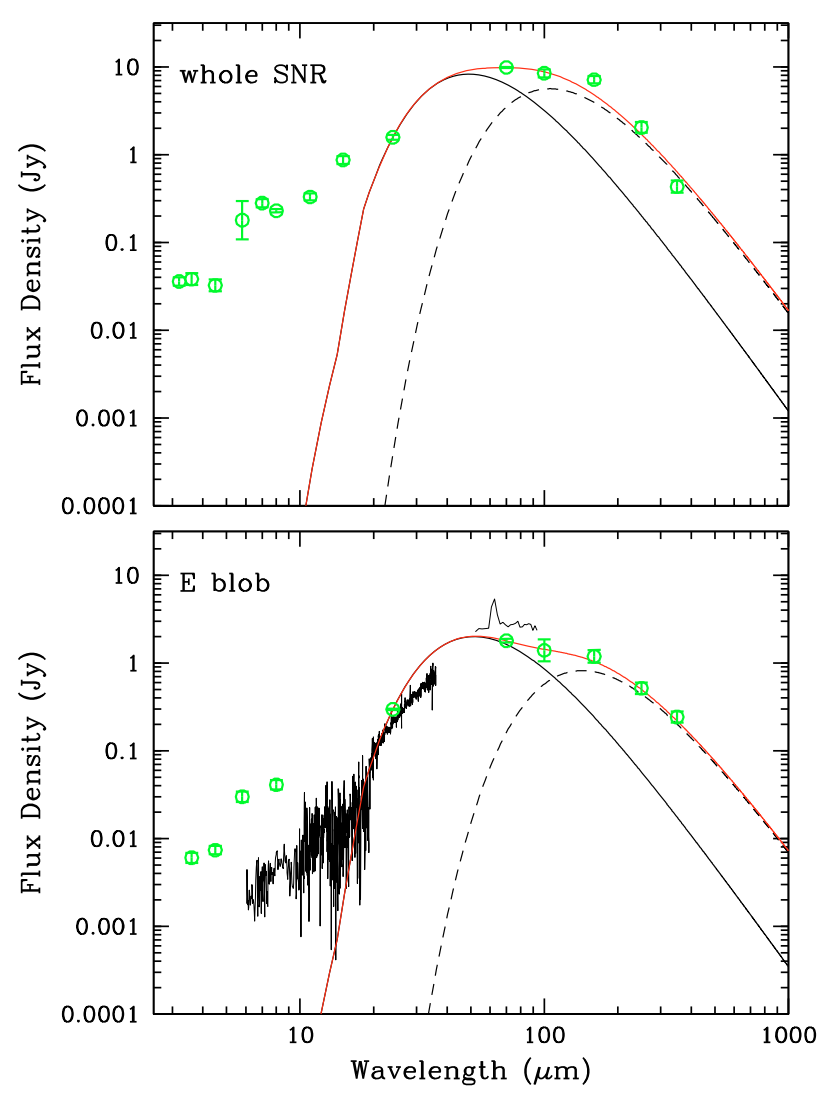

Fig. 2. SED plots of the whole SNR (upper panel) and E blob only (lower panel) together with silicate dust fits. The strong emission lines of atomic gas and $\mathrm{H}_{2}$ in the IRS spectrum are subtracted. The black solid and broken lines are the SEDs of warm and cold components, reflectively. The red lines are the sum of these two components.

Table 2. The derived dust mass and temperature.

\begin{tabular}{lcccc}
\hline \hline Part & $\begin{array}{c}\text { Dust } \\
\text { spices }\end{array}$ & Comp. & $\begin{array}{c}T_{\mathrm{d}} \\
(\mathrm{K})\end{array}$ & $\begin{array}{c}M_{\mathrm{d}} \\
\left(M_{\odot}\right)\end{array}$ \\
\hline SNR & carbon & warm & $62 \pm 2$ & $0.10 \pm 0.02$ \\
& & cold & $26 \pm 2$ & $8.7 \pm 1.0$ \\
& silicate & warm & $57 \pm 2$ & $0.4 \pm 0.06$ \\
& & cold & $27 \pm 2$ & $12 \pm 2$ \\
\hline E blob & carbon & warm & $59 \pm 2$ & $0.04 \pm 0.01$ \\
& & cold & $19 \pm 2$ & $4.2 \pm 1.7$ \\
& silicate & warm & $57 \pm 1$ & $0.10 \pm 0.01$ \\
& & cold & $21 \pm 1$ & $6.1 \pm 2.2$ \\
\hline
\end{tabular}

We first considered a single temperature model to fit the SED. For the whole SNR, the best fit had a $T_{\mathrm{d}}$ of $33 \mathrm{~K}$ and $34 \mathrm{~K}$ for amorphous carbon and for silicate respectively, and a derived $M_{\mathrm{d}}$ of $3.6 M_{\odot}$ and $4.6 M_{\odot}$. For the E blob, $T_{\mathrm{d}}$ is $35 \mathrm{~K}$ (carbon case) and $39 \mathrm{~K}$ (silicate case), and $M_{\mathrm{d}}$ is $0.47 M_{\odot}$ and $0.60 M_{\odot}$. The temperature is fairly similar to the temperature derived by Graham et al. (1987) from IRAS data. However, we found that a better fit could be obtained if we considered a model with twotemperatures instead of one. The one-component fittings cannot simultaneously explain the $<24 \mu \mathrm{m}$ and $>160 \mu \mathrm{m}$ flux. The implied dust masses and temperatures for the two-temperate model are summarized in Table 1 for the two types of grains.

The best-fit two-temperature model, assuming silicate grains, for the SEDs is shown in Fig. 1. The warm component has a temperature of $\sim 60 \mathrm{~K}$, and is surely due to dust in shocked gas, while the cold component has a temperature of $\sim 20 \mathrm{~K}$, similar to that found in molecular clouds 


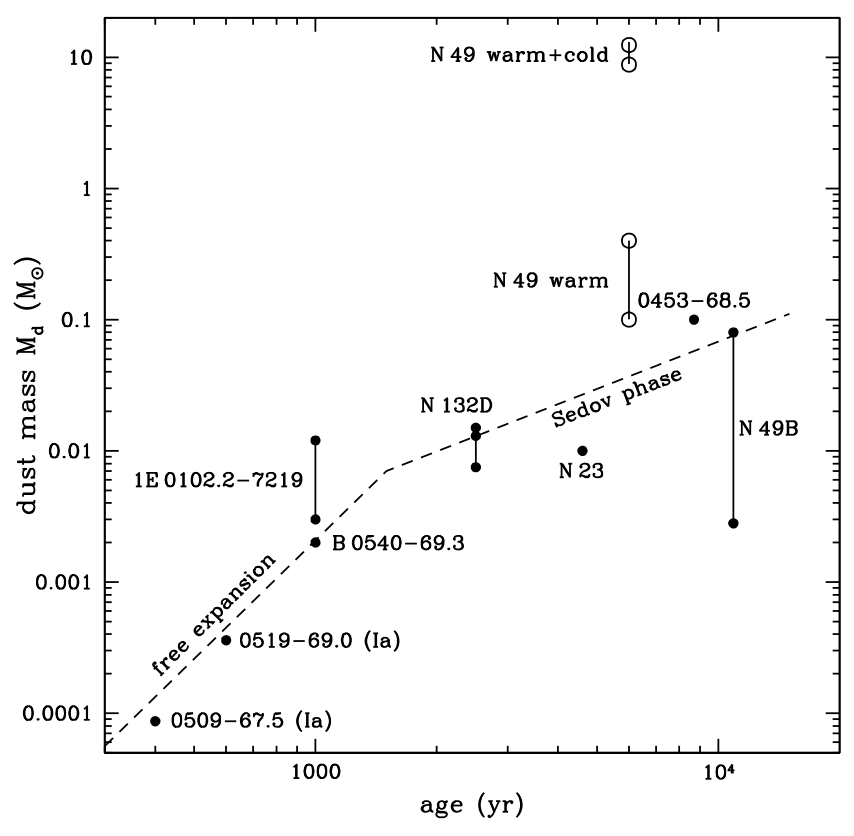

Fig. 3. The relation between the dust mass and age. The data are taken from Seok et al. (2008) for 0509-67.5, 0519-69.0, N132D, and N49B; Williams et al. (2008); Sandstrom et al. (2009) and Rho et al. (2009) for 1E 0102.2-7219; Williams et al. (2006a,b) for N132D, N23, 0453-68.5, and N49B; van Loon et al. (2010) and present work for N49.

(16-24 K, Bernard et al. 2008; Gordon et al. in 2010). The contribution from the cold component to the total flux in the Herschel bands is $>80 \%$. The dust mass and temperature of the warm component in the $\mathrm{E}$ blob are very close to van Loon et al. (2010; $0.2 M_{\odot}$ and $43 \mathrm{~K}$ ). The total mass of dust associated with N49 is about $10 M_{\odot}$ and is dominated by dust at cold temperatures, dust which was difficult to observe with Spitzer. It is tempting therefore to identify the warm component with dust in the X-ray plasma and the cool component not with the SNR, but with the molecular cloud. However, this assignment is very uncertain, because dust temperature variations are also expected as a result of the large variations of post-shock gas density that are known to exist in N49.

To place SNR N49's dust mass estimate into the context of SNR development, we plot the dust mass vs. age for all LMC and SMC SNRs studied to data, as noted in Fig. 3. Note that except for N49 the dust masses are estimated based on $<70 \mu \mathrm{m}$ data. The relation can be with the free expansion phase early on, followed by a Sedov phase (radius $\propto(\text { age })^{2 / 5}$; cf. Badenes et al. 2010).

The mass of the warm dust component in N49 is $0.1 M_{\odot}$ assuming amorphous carbon and $0.4 M_{\odot}$ assuming silicates. Adopting a dust-to-gas mass ratio of 250 (Meixner et al. 2010) and assuming that only the warm dust is associated with shocked gas, the swept-up mass of the SNR ranges from 25 to $100 M_{\odot}$. This is comparable to the mass estimated from X-ray observations, $200 M_{\odot}$ (Hughes et al. 1998), assuming the true grain characteristics are similar to those of silicates. A much higher ( $25 \times$ larger) swept-up mass is estimated if we included the cold dust in our estimate, which suggests that in the context of our simple model there is a significant molecular cloud component to the cold dust we associate with the SNR, and that the shocks propagating into the molecular cloud may be too slow to produce X-rays. While more sophisticated modeling of N49 may refine the mass estimates somewhat, the basic limitation in arriving at a more precise picture of the nature of the interaction with the molecular cloud and the amount of shocked molecular gas in N49 is the limited angular resolution of Herschel at longer wavelengths.

Acknowledgements. We acknowledge financial support from the NASA Herschel Science Center, J.P.L. contract \# 1381522. We are grateful for the contributions and support from the European Space Agency (ESA), the PACS and SPIRE teams, the Herschel Science Center and the NASA Herschel Science Center (esp. A. Barbar and K. Xu) and the PACS and SPIRE instrument control center at CEA-Saclay, without which none of this work would be possible.

\section{References}

Badenes, C., Maoz, D., \& Draine, B. 2010, MNRAS, accepted [arXiv: 1003.3030 ]

Banas, K. R., Hughes, J. P., Bronfman, L., \& Nyman, L.-A. 1997, ApJ, 480, 607 Bernard, J.-Ph., Reach, W. T., Paradis, D., et al. 2008, AJ, 136, 919

Bilikova, J., Williams, R. N. M., Chu, Y.-H., Gruendl, R. A., \& Lundgren, B. F. 2007, AJ, 134, 2308

Cline, T. L., Desai, U. D., Pizzichini, G., et al. 1980, ApJ, 237, L1

Dickel, J. R., \& Milne, D. K. 1998, ApJ, 115, 1057

Draine, B. T., \& Lee, H. M. 1984, ApJ, 285, 89

Gordon, K. D., Galliano, F., Hony, S., et al. 2010, A\&A, 518, L89

Graham, J. R., Evans, A., Albinson, J. S., Bode, M. F., \& Meikle, W. P. S. 1987, ApJ, 319, 126

Hill, R. S., Cheng, K.-P., Bohlin, R. C., et al. 1995, ApJ, 446, 622

Hughes, J. P., Hayashi, I., \& Koyama, K. 1998, ApJ, 505, 732

Kemper, F., et al. 2010, PASP, 122, 892

Kwok, S. 2007, Physics and Chemistry of the Interstellar Medium University Science Books

Long, K. S., Helfand, D. J., \& Grabelsky, D. A. 1981, ApJ, 248, 925

Meixner, M., Gordon, K., Indebetouw, R., et al. 2006, AJ, 132, 2268

Meixner, M., Galliano, F., Hony, S., et al. 2010, A\&A, 518, L71

Morgan, H. L., \& Edmunds, M. G. 2003, MNRAS, 343, 427

Murakami, H., Baba, H., Barthel, P., et al. 2007, PASJ, 59, 369

Onaka, T., Matsuhara, H., Wada, T., et al. 2007, PASJ, 59, 401

Park, S., Burrows, D. N., Garmire, et al. 2003, ApJ, 586, 210

Poglitsch, A., Waelkens, C., Geis, N., et al. 2010, A\&A, 518, L2

Rho, J., Reach, W. T., Tappe, A., et al. 2009, ApJ, 700, 579

Rothschild, R. E., Kulkarni, S. R., \& Lingenfelter, R. E. 1994, Nature, 368, 432 Sandstrom, K. M., Bolatto, A. D., Stanimirović, S., van Loon, J. T., \& Smith, J. D. T. 2009, ApJ, 696, 2138

Schaefer, B. E. 2008, ApJ, 135, 112

Seok, J.Y., Koo, B.-C., Onaka, T., et al. 2008, PASJ, 60, 453

Swinyard, B. M., Ade, P., Baluteau, J.-P., et al. 2010, A\&A, 518, L4

Vancura, O., Blair, W. P., Long, K. S., \& Raymond, J. C. 1992, ApJ, 394, 158

van Loon, J. T, Oliveira, J. M., Gordon, K. D., et al. 2010, AJ, 139, 68

Williams, B. J., Borkowski, K. J., Reynolds, S. P., et al. 2006a, ApJ, 652, L33

Williams, R. M., Chu, Y.-H., \& Gruendl, R. 2006b, AJ, 132, 1877

Williams, B. J., Borkowski, K. J., Reynolds, S. P., et al. 2008, ApJ, 687, 1054

Zubko, V. G., Mennella, V., Colangeli, L., \& Bussoletti, E. 1996, MNRAS, 282, 1321

1 Space Telescope Science Institute, 3700 San Martin Drive, Baltimore, MD 21218, USA

e-mail: otsuka@stsci.edu

2 School of Physical \& Geographical Sciences, Lennard-Jones Laboratories, Keele University, Staffordshire ST5 5BG, UK

3 Department of Physics and Astronomy, University College London, Gower Street, London WC1E 6BT, UK

4 Spitzer Science Center, California Institute of Technology, MS 2206, Pasadena, CA 91125, USA

5 Stratospheric Observatory for Infrared Astronomy, Universities Space Research Association, Mail Stop 211-3, Moffett Field, CA 94035, USA

6 CEA, Laboratoire AIM, Irfu/SAp, Orme des Merisiers, 91191 Gifsur-Yvette, France

7 Steward Observatory, University of Arizona, 933 North Cherry Ave., Tucson, AZ 85721, USA

8 Jodrell Bank Centre for Astrophysics, Alan Turing Building, School of Physics \& Astronomy, University of Manchester, Oxford Road, Manchester M13 9PL, UK

9 Department of Astronomy, Cornell University, Ithaca, NY 14853 , USA 